\title{
Nanoplastic should be better understood
}

\author{
Plastic nanoparticles raise concern because of their potential impact on the environment. However, many questions \\ need to be answered to establish how dangerous they really are.
}

$P$ lastic is everywhere. We only need to look around our houses to gather a small glimpse of how it has become a seemingly essential part of our life. It helps us to preserve food, keep warm, communicate with the world and much more. Sadly, this wonderful material is also at the origin of one of the worst human-created environmental disasters of our times. Like all commodities, plastic is overused and produces waste in amounts of millions of tonnes per year.

Plastic pollution can have a strong impact on the collective imaginary because it has a visual component. It is difficult to remain unaffected by images of sea shores or landfills covered with plastic bags or bottles. Yet the real potential environmental hazard of plastic comes from the minute fragments into which the large specimens break down. Particles smaller than a few millimetres in size are commonly known as microplastics. They have been found to be widespread in the environment, especially in the ocean where they can be ingested by small animals and fish and get stuck in their gut.

There is officially no lower limit to the size of microplastic. However, in the past few years, scientists have started using the term nanoplastic for particles smaller than a few micrometres. More than just a formality, the differentiation is useful because such small particles are very difficult to isolate from their environment with simple methods, such as filtration, that can be used for microplastic. Furthermore, at so small a size, there is a potential risk that instead of being simply stuck in the guts of living organisms, nanoplastic can penetrate tissues much more easily than larger specimens.

Whether this really happens we do not know yet. In fact, the issue with nanoplastic is that we know so little about it, on many fronts. In his Comment, Stephan Wagner explores the open questions that need answering in order to properly evaluate the environmental risks of nanoplastic. First, we simply do not know how much nanoplastic is in the environment. There are analytical methods to study nanoplastic in the laboratory, but they are not necessarily suitable to assess environmental samples, and at this stage we are left with speculation based on what we know about microplastic fragmentation. As mentioned, we can imagine that most nanoplastic results from bigger specimens breaking down, but information on how this

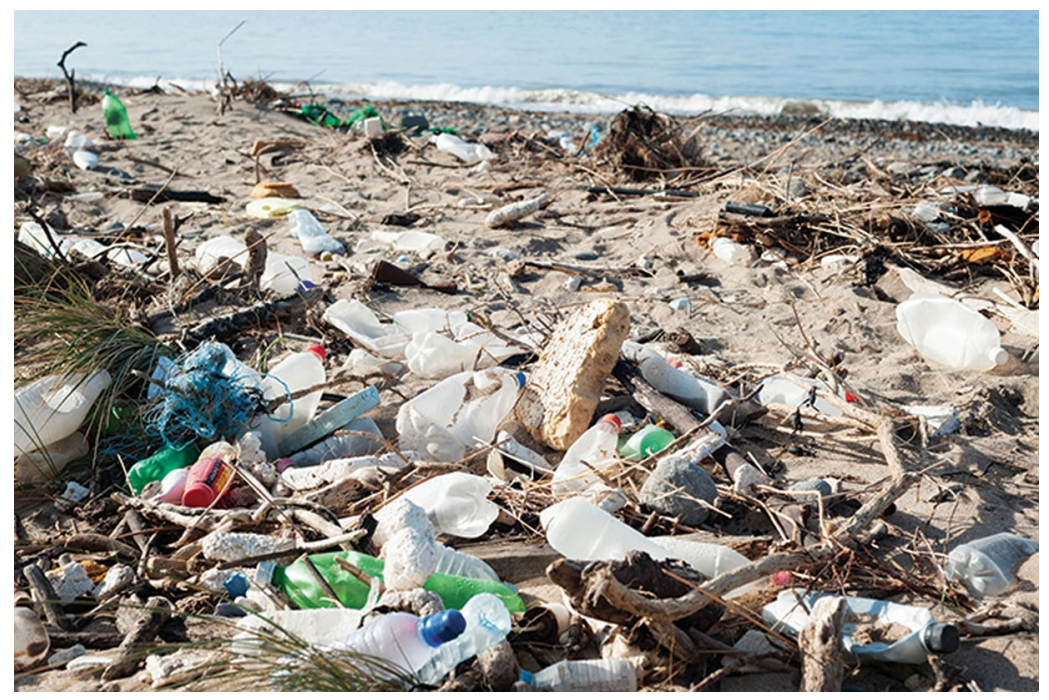

Credit: Daisy Corlett / Alamy Stock Photo

happens is incomplete. Another issue is the environmental fate. Nanoplastic particles come in different shape and compositions, and it is difficult to predict whether they aggregate or sediment and how they transfer through the environment. Finally, there is the question of actual impact. We can imagine that, owing to their small size, nanoplastic particles can penetrate animal tissues and organs, but we do not yet have enough information.

When it comes to monitoring the fate of nanoplastic, a potentially powerful method is presented in a research article by Denise Mitrano and her co-authors. They synthesized plastic nanoparticles with a metallic core that can be traced with analytical techniques commonly available for metals. The results do not solve the problem of monitoring nanoparticles occurring in the environment, but the metal-doped nanoparticles can be seen as proxies for laboratory studies, as highlighted by Albert Koelmans in his News and Views.

In a fourth article in this issue, Michael Sander and co-authors propose using ${ }^{13} \mathrm{C}$-labelled polymers to monitor whether nanoplastic persists in the environment or transforms into its natural components - that is, microbial mass and carbon dioxide. An interesting aspect of this method would be its potential to assess polymer materials labelled as biodegradable and establish whether they simply fragment into small particles undetectable with common techniques or truly degrade into molecular components.

From a nanotechnology perspective, an interesting point that remains unclear is the extent to which, aside from their small size, nanoplastic particles are really nanomaterials. As elegantly explained by Julien Gigault and co-authors (Environ. Pollut. 235, 1030-1034; 2018), whereas engineered nanomaterials are synthesized with the desired size, composition and shape to make use of properties emerging at the nanoscale, nanoplastic is primarily the unwanted product of degradation of larger specimens. So, for example, would the high surface-to-volume ratio affect the way they can absorb other types of pollutants? And would different shapes or surface roughness have an impact on the way in which nanoplastic interacts with biological tissue?

To be clear, Nature Nanotechnology is committed to supporting all studies aimed at establishing the risks posed by nanoscale materials. However, we also feel that microscopic studies of the interaction between plastic nanoparticles and biological tissues would provide essential information on their potential toxicity as well as interesting scientific information.

Published online: 3 April 2019

https://doi.org/10.1038/s41565-019-0437-7 